\title{
EFicácia do Herbicida MSMA na ERRAdicaÇÃo de CoqueIros INFECTADOS COM RESINOSE
}

\author{
Efficacy of the Herbicide MSMA in Eradicating Coconut Trees Infected with Stem-Bleeding \\ FONTES, H.R. ${ }^{2}$, PROCOPIO, S.O. ${ }^{2}$, CARGNELUTTI FILHO, A. ${ }^{3}$, FERREIRA, J.M.S. ${ }^{2}$ e \\ FERNANDES, M.F. ${ }^{2}$
}

\begin{abstract}
RESUMO - Objetivou-se com este trabalho identificar a menor dose do herbicida MSMA e a melhor forma de aplicação, visando à erradicação de coqueiros infectados com resinose. O experimento foi realizado no município de Neópolis-SE, no período de julho a agosto de 2007, utilizando plantas do cultivar Anão-Verde, com 11 anos de idade. O delineamento experimental foi o inteiramente casualizado, com cinco repetições, em esquema fatorial $3 \times 2+1$, sendo três doses do herbicida MSMA $(14,4 ; 21$,6; e 28,8 g por planta), duas formas de aplicação dos tratamentos herbicidas (produto injetado no estipe em um furo de $25 \mathrm{~cm}$ de profundidade e $5 \mathrm{~cm}$ de diâmetro, localizado a 1,0 $\mathrm{m}$ de altura; ou a dose dividida em dois furos de $25 \mathrm{~cm}$ de profundidade e $5 \mathrm{~cm}$ de diâmetro, sendo um a 0,5 $\mathrm{m}$ e o outro a $1,0 \mathrm{~m}$ de altura), mais um tratamento controle sem aplicação. O herbicida foi injetado sem diluição, usando uma seringa graduada, imediatamente após a abertura dos furos. Após a aplicação dos tratamentos, realizou-se o fechamento dos furos com argila umedecida. As avaliações de controle foram realizadas aos $7,14,21$ e 28 dias após a aplicação dos tratamentos. A dose de 21,6 g por planta de MSMA localizada em apenas um furo foi eficiente na erradicação dos coqueiros, proporcionando aos 28 DAA controle acima de 95\%, impedindo qualquer possibilidade de recuperação das plantas.
\end{abstract}

Palavras-chave: Cocos nucifera, Ceratocystis paradoxa, arsenicais orgânicos.

\begin{abstract}
The objective of this work was to identify the minimum MSMA dose needed and the best way to apply it to chemically eradicate coconut palm trees affected by stem-bleeding. The experiment was carried out in Neopolis, Sergipe from July to August 2007. The coconut tree variety green-dwarf had been planted 11 years ago in the area The experiment was laid out in a completely randomized design with five replicates and the treatments were arranged in a $3 \times 2+1$ factorial scheme, with three herbicide doses (14.4, 21.6 and $28.8 \mathrm{~g}$ per plant), two modes of herbicide application (entire dose injection in the stem in a $25 \mathrm{~cm}$ deep $\times 5 \mathrm{~cm}$ diameter hole, located at $1 \mathrm{~m}$ height; or injection of the product splitting the dose between two $25 \mathrm{~cm}$ deep $\times 5 \mathrm{~cm}$ diameter holes, one at $0.5 \mathrm{~m}$ height and the other at $1.0 \mathrm{~m}$ height) plus an additional control treatment with no herbicide application. Undiluted herbicide was applied with a graduated syringe immediately after the drilling of the holes. After herbicide injection, the holes were filled with moist clay. Four coconut eradication evaluations were carried out at 7, 14, 21 and 28 days after application (DAA) of the MSMA treatments. The application of $21.6 \mathrm{~g}$ MSMA per plant, in a single hole, was efficient in eradicating the coconut palm trees, promoting a control above $95 \%$ and precluding any possibility of plant recovery.
\end{abstract}

Keywords: Cocos nucifera, Ceratocystis paradoxa, organic arsenic.

1 Recebido para publicação em 23.7.2008 e na forma revisada em 13.11.2009.

2 Pesquisador da Embrapa Tabuleiros Costeiros, 49025-040 Aracaju-SE, <procopio@cpatc.embrapa.br>; ${ }^{3}$ Professor do Dep. de Fitotecnia da UFSM, 97105-900 Santa Maria-RS. 


\section{INTRODUÇÃO}

Entre os problemas que afetam a cultura do coqueiro (Cocos nucifera) no Estado de Sergipe destacam-se os de ordem fitossanitária. O cultivar plantado, na sua maioria o coqueiro Anão-Verde, tem-se mostrado bastante suscetível a surtos de pragas e doenças em razão da homogeneidade dos indivíduos e da forma de monocultivo com que é explorado (Warwick et al., 2004). A doença que vem resultando em maiores prejuízos é o "stem bleeding" ou resinose, cujo agente causal é o fungo Thielaviopsis paradoxa, Chalara paradoxa ou Ceratocystis paradoxa (Nelson, 2005).

Quando o coqueiro está infectado com essa doença e em grau já avançado, uma das recomendações é a sua erradicação, a fim de se evitar a transmissão da doença, principalmente pelo inseto-vetor Rhynchophorus palmarum (Parra et al., 2003). Esses autores comprovaram que esse inseto carrega em seu tubo digestivo partículas infectivas (conídios) do fungo. A erradicação vem sendo realizada manualmente, por meio do corte do coqueiro, com posterior remoção e queima; todavia, o rendimento operacional tem se mostrado baixo, além de ser prática extremamente onerosa (Ferreira \& Fontes, 2007). Uma alternativa a essa técnica de eliminação mecânica/física pode ser a erradicação química. $O$ uso de herbicidas para controle de espécies arbóreas já vem sendo adotado em pastagens (Carmona et al., 2001), para evitar a rebrota de árvores de interesse econômico após seu corte (Loftis, 1985; Souza et al., 2006), e no controle de espécies utilizadas na produção de narcóticos (Ferreira \& Reddy, 2000).

Vários herbicidas foram preliminarmente testados, sendo o MSMA o composto que apresentou a melhor resposta, tanto em termos de velocidade de ação como no controle final das plantas. O MSMA (metanoarseniato ácido monossódico) é um herbicida pertencente ao grupo dos arsenicais orgânicos, sendo um ácido fraco, com pKa de 4,10 e 9,02, e solubilidade em água a $25^{\circ} \mathrm{C}$ de $1.040 .000 \mathrm{mg} \mathrm{L}^{-1}$ (Vencil, 2002). Seu modo de ação ainda não está bem elucidado, porém sabe-se que provoca destruição das membranas celulares em espécies vegetais sensiveis (Koger et al., 2007). Trabalhos apontam que o MSMA apresenta translocação apoplástica em Sorghum halepense (Kogan \& Araya, 1975), simplástica em Cyperus rotundus (Duble et al., 1969) e apossimplástica em Gossypium hirsutum, Xanthium strumarium (Keese \& Camper, 1994) e Triticum aestivum (Domir et al., 1976).

Uma das vantagens do uso do MSMA na erradicação de coqueiros doentes é que esse xenobiótico praticamente não apresenta ação em pré-emergência (Hiltbold, 1975), ou seja, não deixa resíduos que possam prejudicar um novo plantio da cultura do coco ou de outra espécie vegetal. Trabalhos demonstram que o MSMA também tem efeito inseticida (Del Rivero, 1990), podendo inclusive apresentar efeito tóxico ao principal vetor da resinose: o inseto Rhynchophorus palmarum. Em decorrência disso, o objetivo deste trabalho foi identificar a menor dose do herbicida MSMA e a melhor forma de aplicação, visando à erradicação química de coqueiros infectados com resinose.

\section{MATERIAL E MÉTODOS}

O experimento foi instalado no município de Neópolis - SE $\left(10^{\circ} 19^{\prime}\right.$ de latitude sul, $36^{\circ} 34^{\prime}$ de longitude oeste e $30 \mathrm{~m}$ de altitude), no período de julho a agosto de 2007 . O clima local, pela classificação de Köppen, é do tipo AS' (tropical chuvoso com verão seco), e o solo da área experimental, classificado como Argissolo Amarelo distrófico coeso (Araújo Filho et al., 1999). O coqueiral foi composto pela variedade Anão-Verde, implantada há 11 anos no espaçamento de $7,5 \times 7,5 \times 7,5 \mathrm{~m}$, em triângulo.

O delineamento experimental foi o inteiramente casualizado, com cinco repetições (cada unidade experimental foi composta por um coqueiro), em esquema fatorial $3 \times 2+1$, sendo três doses do herbicida MSMA $(14,4$; 21,6; e 28,8 g por planta), duas formas de aplicação dos tratamentos herbicidas (produto injetado no estipe em um furo de $25 \mathrm{~cm}$ de profundidade e $5 \mathrm{~cm}$ de diâmetro, localizado a $1,0 \mathrm{~m}$ de altura; ou a dose dividida em dois furos de $25 \mathrm{~cm}$ de profundidade e $5 \mathrm{~cm}$ de diâmetro, sendo um a $0,5 \mathrm{~m}$ e o outro a $1,0 \mathrm{~m}$ de altura), mais um tratamento controle (testemunha) sem aplicação. 
A aplicação foi realizada por intermédio de uma seringa graduada imediatamente após a abertura dos furos, sendo o herbicida aplicado sem diluição. Para não haver o vazamento do herbicida aplicado, os furos foram abertos obedecendo a um ângulo de inclinação de $45^{\circ}$. Após a injeção dos tratamentos herbicidas, realizou-se o fechamento dos furos com argila umedecida.

As avaliações visuais da intoxicação das folhas 1,9 e 14 (a contar do ápice), do cacho 14 e da intoxicação geral das plantas foram realizadas aos 7, 14, 21 e 28 dias após a aplicação dos tratamentos (DAA), utilizando-se escala percentual, em que $100 \%$ significa morte da planta, inclusive com queda completa da copa, e $0 \%$ (zero), ausência de sintomas do tratamento herbicida.

Os dados coletados foram submetidos à análise de variância e teste $\mathrm{F}$ a $5 \%$ de significância. A análise dos efeitos significativos das formas de aplicação do herbicida dentro de cada dose do MSMA foi realizada pelo teste de Tukey a 5\% de significância, e o comportamento das doses do herbicida dentro de cada forma de aplicação, por análise de regressão.

\section{RESULTADOS E DISCUSSÃO}

Em todas as avaliações não foi observada interação entre os efeitos das doses de MSMA e a forma de aplicação desse herbicida no estipe dos coqueiros infectados com resinose, sendo observados apenas efeitos isolados.

Inicialmente, verificou-se que a "folha 1" dos coqueiros apresentou os menores niveis de sintomas, mostrando, possivelmente, a maior dificuldade da translocação do herbicida MSMA até esta folha, por estar situada mais próximo do ápice e, consequentemente, mais distante do local de injeção dos produtos. Translocações simplástica e apossimplástica do MSMA foram constatadas por Duble et al. (1969), Domir et al. (1976) e Keese \& Camper (1994). Verificou-se também que a injeção do MSMA concentrada em apenas um furo acelerou a morte da "folha 1" dos coqueiros doentes - fato observado nas avaliações realizadas aos 7 e 14 DAA. Contudo, nas avaliações seguintes (21 e 28 DAA) não mais se detectaram diferenças entre as formas de aplicação desse herbicida (Tabela 1). O incremento nas doses do herbicida MSMA, independentemente da forma de aplicação, resultou em aumento na intoxicação da "folha 1" em todas as avaliações, porém esse aumento apenas foi significativo até a dose de 21,6 g por planta, o que resultou, na última avaliação, em intoxicação média de 93,4\% (Tabela 1 e Figura 1).

A aplicação do MSMA em um único furo no estipe do coqueiro resultou em melhor eficácia na dessecação da "folha 9" apenas aos 14 DAA; nas demais épocas de avaliação não se constataram diferenças significativas entre as duas formas de aplicação de MSMA avaliadas (Tabela 2). $\mathrm{Na}$ avaliação realizada aos 7 DAA, observou-se maior intoxicação da "folha 9" com aumento das doses do MSMA; no entanto, nas avaliações seguintes $(14,21 \mathrm{e}$ 28 DAA) não se observou diferença a partir da dose de 21,6 g por planta (Tabela 2 e Figura 2). Intoxicação média superior a 95\% da "folha 9" foi constatada com a aplicação da dose de 21,6 g por planta, independentemente da forma de aplicação.

Tabela 1 - Intoxicação (\%) da "folha 1" de coqueiros (variedade Anão-Verde) após aplicação de três doses do herbicida MSMA em um ou em dois locais no estipe das plantas. Neópolis-SE, 2007

\begin{tabular}{|c|c|c|c|c|c|c|c|c|c|c|c|c|}
\hline \multirow{3}{*}{ Tratamento herbicida } & \multicolumn{3}{|c|}{$7 \mathrm{DAA}^{*}$} & \multicolumn{3}{|c|}{14 DAA } & \multicolumn{3}{|c|}{21 DAA } & \multicolumn{3}{|c|}{$28 \mathrm{DAA}$} \\
\hline & 1 furo & 2 furos & Média & 1 furo & 2 furos & Média & 1 furo & 2 furos & Média & 1 furo & 2 furos & Média \\
\hline & \multicolumn{12}{|c|}{ Intoxicação (\%) } \\
\hline MSMA $(14,4$ g por planta $)$ & 10,0 & 10,4 & $10,2 \mathrm{~B}$ & 30,0 & 15,6 & $22,8 \mathrm{~B}$ & 41,2 & 43,0 & $42,1 \mathrm{~B}$ & 83,2 & 81,0 & $82,1 \mathrm{~B}$ \\
\hline MSMA (21,6 g por planta) & 25,0 & 14,0 & $19,5 \mathrm{~A}$ & 65,0 & 42,0 & $53,5 \mathrm{~A}$ & 72,8 & 67,6 & $70,2 \mathrm{~A}$ & 93,4 & 93,4 & $93,4 \mathrm{~A}$ \\
\hline MSMA $(28,8$ g por planta $)$ & 24,0 & 20,0 & $22,0 \mathrm{~A}$ & 63,0 & 62,0 & $62,5 \mathrm{~A}$ & 79,6 & 87,6 & $83,6 \mathrm{~A}$ & 92,6 & 96,6 & $94,6 \mathrm{~A}$ \\
\hline Média & $19,7 \mathrm{a}$ & $14,8 \mathrm{~b}$ & - & $52,7 \mathrm{a}$ & $39,9 \mathrm{~b}$ & - & $64,5 \mathrm{a}$ & $66,1 \mathrm{a}$ & - & $89,7 \mathrm{a}$ & $90,3 \mathrm{a}$ & - \\
\hline
\end{tabular}

Médias seguidas pelas mesmas letras, minúsculas na linha (horizontal) e maiúsculas na coluna (vertical), não diferem estatisticamente entre si pelo teste de Tukey a $5 \%$. ${ }^{*} \mathrm{DAA}=$ dias após a aplicação do herbicida. 
Das folhas do coqueiro avaliadas, a "folha 14", a mais velha, apresentou maior intoxicação. Biberson \& Duhamel (1987), após injeção do MSMA no estipe de coqueiros, também verificaram morte das folhas mais velhas antes das mais novas, concordando com os resultados observados. Como verificado para a "folha 9", somente aos 14 DAA é que se observou maior efeito da aplicação do MSMA localizada em um único furo do estipe do coqueiro na intoxicação da "folha 14" (Tabela 3).

O fato de a aplicação poder ser realizada em apenas um furo resulta em aumento no rendimento operacional, já que a abertura do furo é a operação que mais demanda tempo no processo de erradicação química de

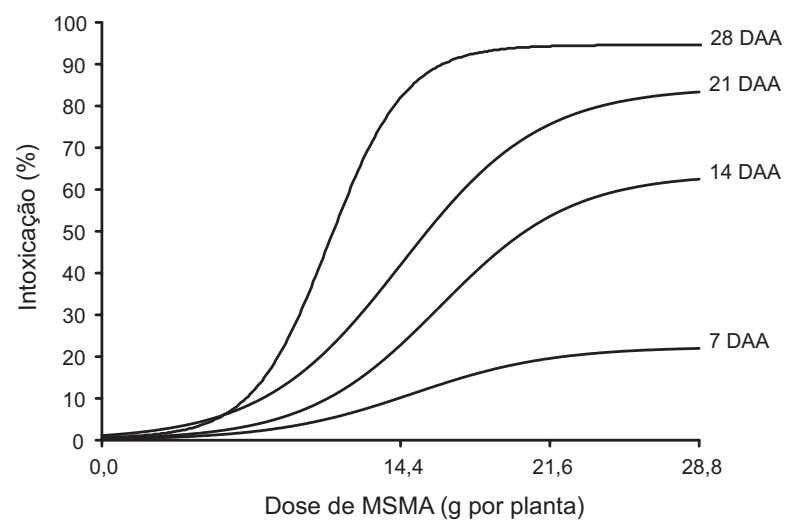

DAA $=$ dias após a aplicação do herbicida. Equações de regressão: 7 DAA - $\hat{\mathrm{Y}}=22,3182 /(1+\exp (-(\mathrm{X}-15,0025) / 3,3634)), \mathrm{R}^{2}=0,99$; $14 \mathrm{DAA}-\hat{\mathrm{Y}}=63,7283 /(1+\exp (-(\mathrm{X}-16,2821) / 3,2042)), \mathrm{R}^{2}=0,99$; $21 \mathrm{DAA}-\hat{\mathrm{Y}}=84,4745 /(1+\exp (-(\mathrm{X}-14,4510) / 3,3417)), \mathrm{R}^{2}=0,99$; 28 DAA $-\hat{\mathrm{Y}}=94,1476 /(1+\exp (-(\mathrm{X}-10,8788) / 1,8365)), \mathrm{R}^{2}=0,99$.

Figura 1 - Intoxicação da "folha 1" de coqueiros (variedade Anão-Verde) após aplicação de diferentes doses do herbicida MSMA no estipe das plantas. Neópolis-SE, 2007. coqueiros infectados com resinose. A dose de 28,8 g por planta de MSMA proporcionou aos 7 DAA incremento na intoxicação da "folha 14", mas nas avaliações realizadas aos 14 e 21 DAA não mais diferiu da dose de $21,6 \mathrm{~g}$ por planta. No entanto, aos 28 DAA observou-se progresso significativo da intoxicação da "folha 14" dos coqueiros que receberam a menor dose do MSMA $(14,4 \mathrm{~g}$ por planta), a ponto de não diferir da dessecação verificada com a aplicação das maiores doses (21,6 e 28,8 g por planta), que proporcionaram seca completa das folhas, culminando na queda destas (Tabela 3 e Figura 3).

A intoxicação do "cacho 14" dos coqueiros apenas foi influenciada pela forma de aplicação

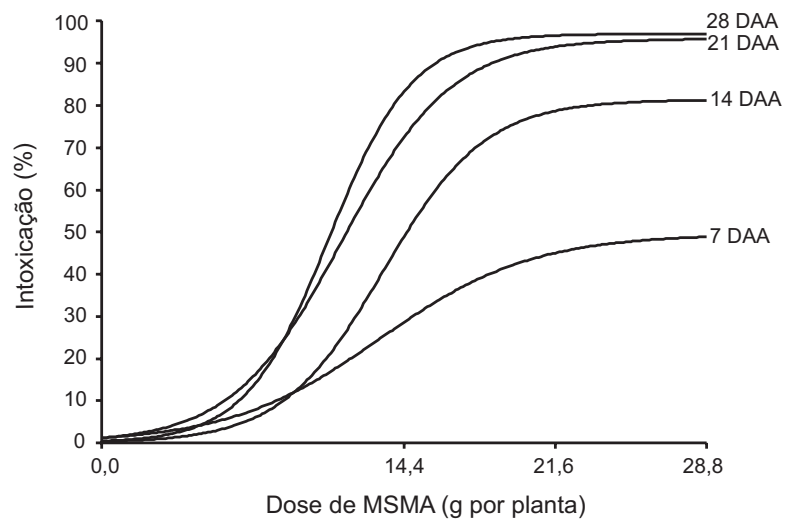

DAA = dias após a aplicação do herbicida. Equações de regressão 7 DAA - $\hat{\mathrm{Y}}=49,5097 /(1+\exp (-(\mathrm{X}-13,2681) / 3,6115)), \mathrm{R}^{2}=0,99$; 14 DAA $-\hat{\mathrm{Y}}=81,3658 /(1+\exp (-(\mathrm{X}-13,4095) / 2,4231)), \mathrm{R}^{2}=0,99$; $21 \mathrm{DAA}-\hat{\mathrm{Y}}=95,8111 /(1+\exp (-(\mathrm{X}-11,4480) / 2,6058)), \mathrm{R}^{2}=0,99$; 28 DAA $-\hat{\mathrm{Y}}=96,9883 /(1+\exp (-(\mathrm{X}-10,8201) / 1,9866)), \mathrm{R}^{2}=0,99$.

Figura 2 - Intoxicação da "folha 9" de coqueiros (variedade Anão-Verde) após aplicação de diferentes doses do herbicida MSMA no estipe das plantas. Neópolis-SE, 2007.

Tabela 2 - Intoxicação (\%) da "folha 9" de coqueiros (variedade Anão-verde) após aplicação de três doses do herbicida MSMA em um ou em dois locais no estipe das plantas. Neópolis-SE, 2007

\begin{tabular}{|c|c|c|c|c|c|c|c|c|c|c|c|c|}
\hline \multirow{3}{*}{ Tratamento herbicida } & \multicolumn{3}{|c|}{$7 \mathrm{DAA}^{*}$} & \multicolumn{3}{|c|}{$14 \mathrm{DAA}$} & \multicolumn{3}{|c|}{$21 \mathrm{DAA}$} & \multicolumn{3}{|c|}{$28 \mathrm{DAA}$} \\
\hline & 1 furo & 2 furos & Média & 1 furo & 2 furos & Média & 1 furo & 2 furos & Média & 1 furo & 2 furos & Média \\
\hline & \multicolumn{12}{|c|}{ Intoxicação (\%) } \\
\hline MSMA $(14,4 \mathrm{~g}$ por planta $)$ & 28,8 & 29,2 & $29,0 \mathrm{C}$ & 53,8 & 44,0 & $48,9 \mathrm{~B}$ & 77,4 & 68,0 & $72,7 \mathrm{~B}$ & 85,0 & 81,6 & $83,3 \mathrm{~B}$ \\
\hline MSMA (21,6 g por planta) & 41,2 & 41,0 & $41,1 \mathrm{~B}$ & 86,2 & 71,0 & $78,6 \mathrm{~A}$ & 95,0 & 89,6 & $92,3 \mathrm{~A}$ & 97,4 & 94,0 & $95,7 \mathrm{~A}$ \\
\hline $\operatorname{MSMA}(28,8$ g por planta $)$ & 49,0 & 50,0 & $49,5 \mathrm{~A}$ & 79,6 & 83,0 & $81,3 \mathrm{~A}$ & 96,0 & 98,2 & $97,1 \mathrm{~A}$ & 97,4 & 98,2 & $97,8 \mathrm{~A}$ \\
\hline Média & $39,7 \mathrm{a}$ & $40,1 \mathrm{a}$ & - & $73,2 \mathrm{a}$ & $66,0 \mathrm{~b}$ & - & $89,5 \mathrm{a}$ & $85,3 \mathrm{a}$ & - & $93,3 \mathrm{a}$ & $91,3 \mathrm{a}$ & - \\
\hline
\end{tabular}

Médias seguidas pelas mesmas letras, minúsculas na linha (horizontal) e maiúsculas na coluna (vertical), não diferem estatisticamente entre si pelo teste de Tukey a $5 \%$. ${ }^{\mathrm{D}} \mathrm{DAA}=$ dias após a aplicação do herbicida. 
do herbicida MSMA na avaliação realizada aos 14 DAA, quando se constatou que concentrar todo o produto em um único furo no estipe resultou em maior eficácia (Tabela 4). Nas três últimas avaliações $(14,21$ e 28 DAA) verificouse similaridade de resposta entre as doses de 21,6 e 28,8 g por planta, que foram superiores à resultante da aplicação da dose de $14,4 \mathrm{~g}$ por planta. Contudo, na avaliação final realizada aos 28 DAA, não mais se detectaram diferenças entre as doses do MSMA, sendo todas as doses avaliadas eficientes, causando a morte dessa infrutescência (Tabela 4 e Figura 4).

Em relação à avaliação que considerou o aspecto geral das plantas, aos 7 DAA verificouse aumento da intoxicação dos coqueiros com o incremento das doses do MSMA, chegando a atingir $53,0 \%$ na maior dose ( $28,8 \mathrm{~g}$ por planta) (Tabela 5). No entanto, nessa avaliação não se observaram diferenças entre as formas de aplicação. Nas três avaliações seguintes (14, 21 e 28 DAA) não se detectaram diferenças entre as doses de 21,6 e $28,8 \mathrm{~g}$ por planta, porém estas doses resultaram em maior intoxicação em relação à dose de 14,4 g por planta.
Biberson \& Duhamel (1987), em trabalho realizado no intuito de erradicar quimicamente coqueiros sadios, constataram que o uso do

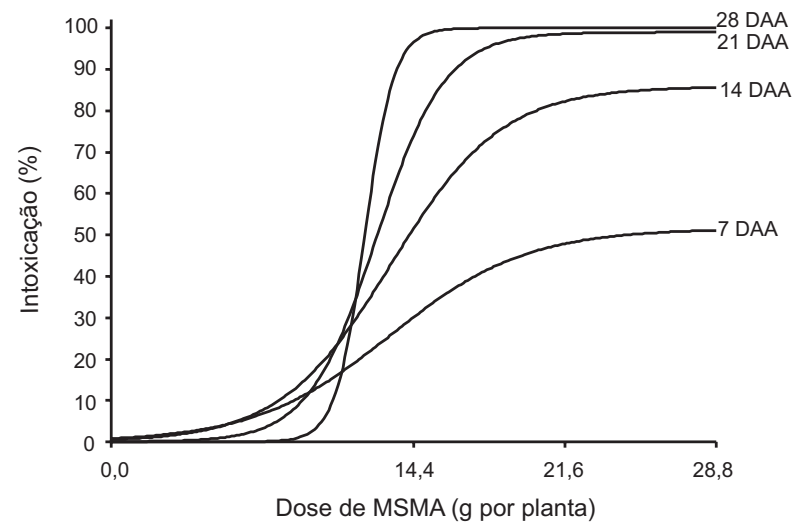

DAA $=$ dias após a aplicação do herbicida. Equações de regressão: $7 \mathrm{DAA}-\hat{\mathrm{Y}}=51,5571 /(1+\exp (-(\mathrm{X}-13,2991) / 3,2323)), \mathrm{R}^{2}=0,99$; 14 DAA - $\hat{\mathrm{Y}}=85,8781 /(1+\exp (-(\mathrm{X}-13,3262) / 2,6395)), \mathrm{R}^{2}=0,99$; $21 \mathrm{DAA}-\hat{\mathrm{Y}}=98,9964 /(1+\exp (-(\mathrm{X}-12,6450) / 1,6171)), \mathrm{R}^{2}=0,99$; $28 \mathrm{DAA}-\hat{\mathrm{Y}}=100,0000 /(1+\exp (-(\mathrm{X}-12,0778) / 0,6939)), \mathrm{R}^{2}=1,00$.

Figura 3 - Intoxicação da "folha 14" de coqueiros (variedade Anão-Verde) após aplicação de diferentes doses do herbicida MSMA no estipe das plantas. Neópolis-SE, 2007.

Tabela 3 - Intoxicação (\%) da "folha 14" de coqueiros (variedade Anão-Verde) após aplicação de três doses do herbicida MSMA em um ou em dois locais no estipe das plantas. Neópolis-SE, 2007

\begin{tabular}{|c|c|c|c|c|c|c|c|c|c|c|c|c|}
\hline \multirow{3}{*}{ Tratamento herbicida } & \multicolumn{3}{|c|}{7 DAA* } & \multicolumn{3}{|c|}{14 DAA } & \multicolumn{3}{|c|}{$21 \mathrm{DAA}$} & \multicolumn{3}{|c|}{$28 \mathrm{DAA}$} \\
\hline & 1 furo & 2 furos & Média & 1 furo & 2 furos & Média & 1 furo & 2 furos & Média & 1 furo & 2 furos & Média \\
\hline & \multicolumn{12}{|c|}{ Intoxicação (\%) } \\
\hline MSMA $(14,4$ g por planta) & 9,4 & 16,0 & $12,7 \mathrm{~B}$ & 47,4 & 35,0 & $41,2 \mathrm{~B}$ & 80,0 & 77,0 & $78,5 \mathrm{~B}$ & 99,2 & 96,0 & $97,6 \mathrm{~A}$ \\
\hline MSMA (21,6 g por planta) & 22,4 & 19,0 & $20,7 \mathrm{AB}$ & 74,8 & 50,0 & $62,4 \mathrm{~A}$ & 100,0 & 96,0 & $98,0 \mathrm{~A}$ & 100,0 & 100,0 & $100,0 \mathrm{~A}$ \\
\hline MSMA $(28,8 \mathrm{~g}$ por planta $)$ & 22,0 & 23,6 & $22,8 \mathrm{~A}$ & 74,0 & 72,0 & $73,0 \mathrm{~A}$ & 98,0 & 100,0 & $99,0 \mathrm{~A}$ & 100,0 & 100,0 & $100,0 \mathrm{~A}$ \\
\hline Média & $17,9 \mathrm{a}$ & $19,5 \mathrm{a}$ & - & $65,4 \mathrm{a}$ & $52,3 \mathrm{~b}$ & - & $92,7 \mathrm{a}$ & $91,0 \mathrm{a}$ & - & $99,7 \mathrm{a}$ & $98,7 \mathrm{a}$ & - \\
\hline
\end{tabular}

Médias seguidas pelas mesmas letras, minúsculas na linha (horizontal) e maiúsculas na coluna (vertical), não diferem estatisticamente entre si pelo teste de Tukey a 5\%. ${ }^{*} \mathrm{DAA}=$ dias após a aplicação do herbicida.

Tabela 4 - Intoxicação (\%) do "cacho 14" de coqueiros (variedade anão-verde) após aplicação de três doses do herbicida MSMA em um ou em dois locais no estipe das plantas. Neópolis-SE. 2007

\begin{tabular}{|c|c|c|c|c|c|c|c|c|c|c|c|c|}
\hline \multirow{3}{*}{ Tratamento herbicida } & \multicolumn{3}{|c|}{$7 \mathrm{DAA}^{*}$} & \multicolumn{3}{|c|}{14 DAA } & \multicolumn{3}{|c|}{21 DAA } & \multicolumn{3}{|c|}{28 DAA } \\
\hline & 1 furo & 2 furos & Média & 1 furo & 2 furos & Média & 1 furo & 2 furos & Média & 1 furo & 2 furos & Média \\
\hline & \multicolumn{12}{|c|}{ Intoxicação (\%) } \\
\hline MSMA $(14,4 \mathrm{~g}$ por planta $)$ & 9,4 & 16,0 & $12,7 \mathrm{~B}$ & 47,4 & 35,0 & $41,2 \mathrm{~B}$ & 80,0 & 77,0 & $78,5 \mathrm{~B}$ & 99,2 & 96,0 & $97,6 \mathrm{~A}$ \\
\hline MSMA $(21,6$ g por planta $)$ & 22,4 & 19,0 & $20,7 \mathrm{AB}$ & 74,8 & 50,0 & $62,4 \mathrm{~A}$ & 100,0 & 96,0 & $98,0 \mathrm{~A}$ & 100,0 & 100,0 & $100,0 \mathrm{~A}$ \\
\hline MSMA $(28,8 \mathrm{~g}$ por planta $)$ & 22,0 & 23,6 & $22,8 \mathrm{~A}$ & 74,0 & 72,0 & $73,0 \mathrm{~A}$ & 98,0 & 100,0 & $99,0 \mathrm{~A}$ & 100,0 & 100,0 & $100,0 \mathrm{~A}$ \\
\hline Média & $17,9 \mathrm{a}$ & $19,5 \mathrm{a}$ & - & $65,4 \mathrm{a}$ & $52,3 \mathrm{~b}$ & - & $92,7 \mathrm{a}$ & $91,0 \mathrm{a}$ & - & $99,7 \mathrm{a}$ & $98,7 \mathrm{a}$ & - \\
\hline
\end{tabular}

Médias seguidas pelas mesmas letras, minúsculas na linha (horizontal) e maiúsculas na coluna (vertical), não diferem estatisticamente entre si pelo teste de Tukey a 5\%. ${ }^{*} \mathrm{DAA}=$ dias após a aplicação do herbicida. 


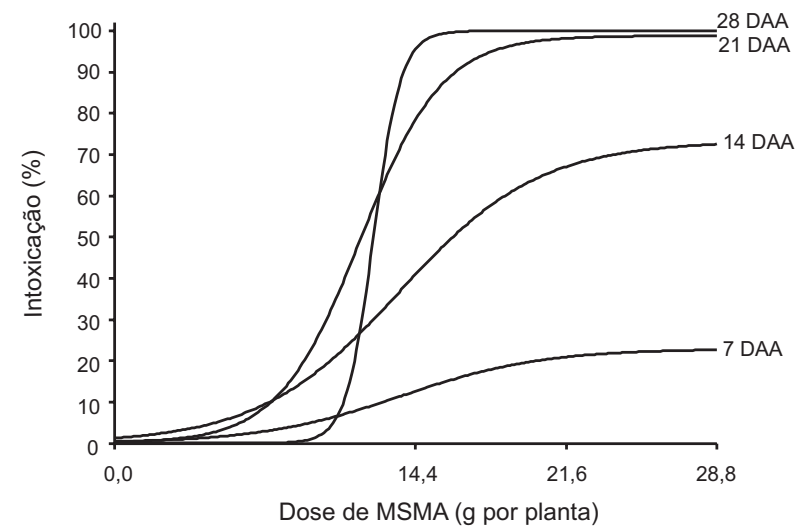

DAA $=$ dias após a aplicação do herbicida. Equações de regressão: 7 DAA - $\hat{\mathrm{Y}}=22,9145 /(1+\exp (-(\mathrm{X}-13,7160) / 3,3706)), \mathrm{R}^{2}=0,99$; 14 DAA $-\hat{\mathrm{Y}}=73,2963 /(1+\exp (-(\mathrm{X}-13,6132) / 3,3582)), \mathrm{R}^{2}=0,99$; 21 DAA $-\hat{\mathrm{Y}}=98,8332 /(1+\exp (-(\mathrm{X}-11,7597) / 1,9555)), \mathrm{R}^{2}=0,99$; $28 \mathrm{DAA}-\hat{\mathrm{Y}}=100,0000 /(1+\exp (-(\mathrm{X}-12,3893) / 0,6531)), \mathrm{R}^{2}=1,00$.

Figura 4 - Intoxicação do "cacho 14" de coqueiros (variedade Anão-Verde) após aplicação de diferentes doses do herbicida MSMA no estipe das plantas. Neópolis-SE, 2007.

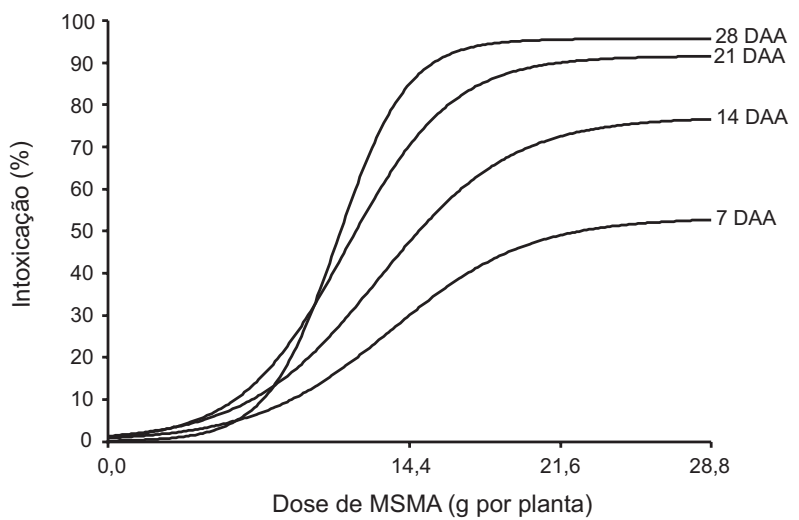

DAA $=$ dias após a aplicação do herbicida. Equações de regressão 7 DAA - $\hat{\mathrm{Y}}=53,1722 /(1+\exp (-(\mathrm{X}-13,5496) / 3,2587)), \mathrm{R}^{2}=0,99$; $14 \mathrm{DAA}-\hat{\mathrm{Y}}=77,1088 /(1+\exp (-(\mathrm{X}-12,9037) / 3,1583)), \mathrm{R}^{2}=0,99$; $21 \mathrm{DAA}-\hat{\mathrm{Y}}=91,6051 /(1+\exp (-(\mathrm{X}-11,3729) / 2,5216)), \mathrm{R}^{2}=0,99$; $28 \mathrm{DAA}-\hat{\mathrm{Y}}=95,6821 /(1+\exp (-(\mathrm{X}-10,9577) / 1,6601)), \mathrm{R}^{2}=0,99$

Figura 5 - Intoxicação de coqueiros (variedade Anão-Verde) após aplicação de diferentes doses do herbicida MSMA no estipe das plantas. Neópolis-SE, 2007

Tabela 5 - Intoxicação (\%) de coqueiros (variedade Anão-verde) após aplicação de três doses do herbicida MSMA em um ou em dois locais no estipe das plantas. Neópolis-SE, 2007

\begin{tabular}{|c|c|c|c|c|c|c|c|c|c|c|c|c|}
\hline \multirow{3}{*}{ Tratamento herbicida } & \multicolumn{3}{|c|}{$7 \mathrm{DAA}^{*}$} & \multicolumn{3}{|c|}{14 DAA } & \multicolumn{3}{|c|}{$21 \mathrm{DAA}$} & \multicolumn{3}{|c|}{$28 \mathrm{DAA}$} \\
\hline & 1 furo & 2 furos & Média & 1 furo & 2 furos & Média & 1 furo & 2 furos & Média & 1 furo & 2 furos & Média \\
\hline & \multicolumn{12}{|c|}{ Intoxicação (\%) } \\
\hline MSMA (14,4 g por planta) & 31,4 & 29,0 & $30,2 \mathrm{C}$ & 51,0 & 44,6 & $47,8 \mathrm{~B}$ & 74,4 & 66,8 & $70,6 \mathrm{~B}$ & 85,8 & 84,2 & $85,0 \mathrm{~B}$ \\
\hline MSMA (21,6 g por planta) & 40,0 & 43,2 & $41,6 \mathrm{~B}$ & 79,2 & 64,0 & $71,6 \mathrm{~A}$ & 90,8 & 86,4 & $88,6 \mathrm{~A}$ & 96,8 & 93,8 & $95,3 \mathrm{~A}$ \\
\hline MSMA (28,8 g por planta) & 52,4 & 53,6 & $53,0 \mathrm{~A}$ & 78,0 & 76,6 & $77,3 \mathrm{~A}$ & 90,4 & 95,2 & $92,8 \mathrm{~A}$ & 95,4 & 96,4 & $95,9 \mathrm{~A}$ \\
\hline Média & $41,3 \mathrm{a}$ & $41,9 \mathrm{a}$ & - & $69,4 \mathrm{a}$ & $61,7 \mathrm{~b}$ & - & $85,2 \mathrm{a}$ & $82,8 \mathrm{a}$ & - & $92,7 \mathrm{a}$ & $91,5 \mathrm{a}$ & - \\
\hline
\end{tabular}

Médias seguidas pelas mesmas letras, minúsculas na linha (horizontal) e maiúsculas na coluna (vertical), não diferem estatisticamente entre si pelo teste de Tukey a 5\%. *DAA = dias após a aplicação do herbicida

MSMA é viável para a operacionalização dessa técnica, recomendando a dose de $25 \mathrm{~g}$ por planta do herbicida. Apenas constatou-se efeito significativo entre as formas de aplicação na avaliação realizada aos $14 \mathrm{DAA}$, em que se verificou que a aplicação do herbicida concentrada em apenas um furo teve melhor efeito que a divisão da dose em dois locais no estipe (Figura 5).

A aplicação da dose de 21,6 g por planta de MSMA localizada em apenas um furo foi eficiente na erradicação dos coqueiros, proporcionando aos 28 DAA controle acima de $95 \%$, impedindo qualquer possibilidade de recuperação das plantas.

\section{LITERATURA CITADA}

ARAÚJO FILHO, J. C. et al. Levantamento de reconhecimento de média intensidade dos solos da região dos tabuleiros costeiros e da baixada litorânea do Estado de Sergipe. Rio de Janeiro: Embrapa Solos, 1999. (Boletim de Pesquisa)

BIBERSON, O.; DUHAMEL, G. Empoisonnement des cocotiers au MSMA (methylarsonate monsodique)

Oleagineux, v. 42 , n. 10 , p. $363-367,1987$.

CARMONA, R.; ARAÚJO NETO, B. S. C.; PEREIRA, R. C. Controle de Acacia farnesiana e de Mimosa pteridofita em pastagem. Pesq. Agropec. Bras., v. 36, n. 10, p. 1301-1307, 2001. 
DEL RIVERO, J. M. Investigacion sobre otros efectos plaguicidas potenciales diferentes de varios herbicidas; especialmente bipiridilos y organo arsenicales. B. Sanidad Veg. Plagas, v. 16, n. 3, p. 605-611, 1990.

DOMIR, S. C. et al. Translocation and metabolic fate of monosodium methanearsonic acid in wheat (Triticum aestivum L.). J. Agric. Food Chem., v. 24, n. 6, p. 1214-1217, 1976.

DUBLE, R. L.; HOLT, E. C.; McBEE, G. G. Translocation and break-down of disodium methanearsonate (DSMA) in coastal bermudagrass. J. Agric. Food Chem., v. 17, n. 6, p. $1247-1250,1969$

FERREIRA, J. F. S.; REDDY, K. N. Absorption and translocation of glyphosate in Erythroxylum coca and E. novogranatense. Weed Sci., v. 48, n. 2, p. 193-199, 2000 .

FERREIRA, J. M. S.; FONTES, H. R. Relatório sobre o atual estágio da doença resinose no Distrito de Irrigação Platô de Neópolis. Aracaju: Embrapa Tabuleiros Costeiros, 2007. $22 \mathrm{p}$

HILTBOLD, A. E. Behaviour of organoarsenicals in plants and soils. In: WOOLSON, E.A. (Ed.). Arsenical pesticides Washington: American Chemical Society Symposium, 1975. v. 7. p. $53-69$.

KEESE, R. J.; CAMPER, N. D. Uptake and translocation of [14C] MSMA in cotton and MSMA-resistant and susceptible cocklebur. Pestic. Biochem. Physiol., v. 49, n. 1, p. $138-145,1994$.
KOGAN, M:; ARAYA, A. Translocacion del metil arsonato monosodico (MSMA) en maicillo (Sorghum halepense (L.) Pers.) y su efecto en la produccion de rizomas y raices. Inv. Agric., v. 1, n. 1, p. 48-53, 1975.

KOGER, C. H. et al. MSMA antagonizes glyphosate and glufosinate efficacy on broadleaf and grass weeds. Weed Technol., v. 21, n. 1, p. 159-165, 2007.

LOFTIS, D. L. Preharvest herbicide treatment improves regeneration in Southern Appalachian Hardwoods. Southern J. Applied For., v. 9, n. 1, p. 177-180, 1985.

NELSON, S. Stem bleeding of coconut palm. Plant Disease, St. Paul, set. 2005. Disponível em: <http:// www.ctahr.hawaii.edu/oc/freepubs/pdf/PD-30.pdf $>$. Acesso em: 11 dez. 2007.

PARRA, D. et al. Presencia de Thielaviopsis paradoxa De Seynes Höhn en el tubo digestivo de Rhynchophorus palmarum Linneo (Coleoptera: Curculionidae).

Entomotropica, v. 18, n. 1, p. 49-55, 2003.

SOUZA, G. V. R. et al. Exsudato radicular de imazapyr aplicado sobre mudas de diferentes clones de eucalipto. Planta Daninha, v. 24, n. 1, p. 141-147, 2006.

VENCIL, W. K. Herbicide handbook. 8.ed. Lawrence: Weed Science Society of America, 2002. 493 p.

WARWICK, D. R. N.; FERREIRA, J. M. S.; PASSOS, E. E $\mathrm{M}$. Ocorrência de resinose do estipe do coqueiro em Sergipe provocada por Chalara paradoxa. Fitopatol. Bras., v. 29, Suplemento, p. 171, 2004. 